\title{
3 The Principle of the Common Cause and the Bell Inequalities
}

In this chapter we will discuss the famous Bell inequalities and whether the fact they are falsified both by the predictions of quantum mechanics and by empirical tests impugns the Principle of the Common Cause, and, if so, which version of it is in danger.

\subsection{Towards the Derivations of the Inequalities}

\subsubsection{Introduction}

Consider a source emitting pairs of spin- $\frac{1}{2}$ particles prepared in the singlet state $\frac{1}{\sqrt{2}}(|\uparrow \downarrow\rangle-|\downarrow \uparrow\rangle)$. Assume that each particle travels towards one of two spatially separated detectors (which we will label "L" and "R"). During the flight of the particles each detector is set to measure the spin of the particle in a certain direction. The detectors are situated so that light emitted on measurement at one detector cannot reach the location of the remaining detector before the other measurement takes place. Assume there is a finite set of possible detector settings. The result of the measurement is always binary-"up" or "down"1, which we will refer to by "+" and "-”. From the formalism of quantum mechanics it follows that the probability of obtaining a "+" result on both particles is equal to $\frac{1}{2} \sin ^{2}\left(\frac{\phi_{i j}}{2}\right)$, where $\phi_{i j}$ is the angle between the direction $i$, set on the left detector, and direction $j$, set on the right detector. And so, if the directions are identical, a perfect anticorrelation of results is expected-a "+" from the left detector means a "-" from the right one. On the other hand, the probability of obtaining a "+" from a detector is predicted to be $\frac{1}{2}$, regardless of the setting. Since the joint probabilities are not, in general, equal to $\frac{1}{4}$, there will be correlations between the results. The stunning result of Bell (1964) is that if a hidden variable (e.g. the complete state of the source) is posited as screening off the results, it is possible (with some additional intuitive assumptions) to derive inequalities falsified by the above predictions. This has been subsequently corroborated experimentally (see the classical paper Aspect et al. (1982) or, for newer results, Scheidl et al. (2010)).

The exposition in the last paragraph was necessarily informal, since we did not want to settle in advance the formalism in which the Bell inequalities are to be formulated and discussed. This is due to the fact that there are two approaches present in the literature, frequently called "big space” and "many spaces approach". Since the

1 We do not include particles which are emitted by the source but do not hit any of the detectors in the picture. There are models for Bell-type correlations which exploit the inefficiency of detectors-see section 3.3 . 
PCC in various formulations considers the existence of events in probability spaces, to judge the force with which the violation of Bell inequalities strikes the PCC we have to be clear how the probability spaces involved look like.

\subsubsection{The Big Space Approach and the Many Spaces Approach}

Consider a probability space $\langle\Omega, \mathcal{F}, P\rangle$. Any event $A \in \mathcal{F}$ with non-trivial probability ${ }^{2}$ induces a new measure on the same event space: for any $C \in \mathcal{F}, P_{A}(C):=P(C \mid A)$. In fact, a "smaller" probability space is induced: $\left\langle A, \mathcal{F}_{A}, P_{A}\right\rangle$, where $\mathcal{F}_{A}:=\{C \cap A \mid C \in \mathcal{F}\}$. In short, an $n$-element partition of the sample space induces $n$ "smaller" probability spaces, provided that each element of the partition has positive probability. The probability of an event in one of the smaller spaces is interpreted as conditional probability in the original "big" space.

In the other direction, suppose you have two probability spaces $\left\langle\Omega_{1}, \mathcal{F}_{1}, P_{1}\right\rangle$ and $\left\langle\Omega_{2}, \mathcal{F}_{2}, P_{2}\right\rangle$. You can then build a "bigger" probability space. First, take as the new sample space $\Omega$ the Cartesian product of $\Omega_{1}$ and $\Omega_{2}$. Then consider the set of "rectangles", that is, sets of the shape $A_{1} \times A_{2}$ for some $A_{1} \in \mathcal{F}_{1}, A_{2} \in \mathcal{F}_{2}$. The first task, before the set of rectangles is expanded to be a proper event space, is to define the measure $P$ on it. And the only requirement is that it should have the so called "marginal property"; that is, for any $A \in \mathcal{F}_{1}, P\left(A \times \Omega_{2}\right)$ should be equal to $P_{1}(A)$ and similarly $P\left(\Omega_{1} \times B\right)$ should be equal to $P_{2}(B)$ for any $B \in \mathcal{F}_{2}$. It is due to this requirement that each of the smaller spaces is embeddable in the big one. But it is easy to see that there is in general more than one way of defining the measure $P$ on the set of rectangles, and therefore, one cannot speak of "the" big space constructed from the smaller spaces.

A different, more informal, but perhaps more illuminating difficulty concerns assigning weights to alternatives. Suppose you have a fair coin and can either toss it (with probabilities $P_{A}(H)=P_{A}(T)=\frac{1}{2}$ ), or conduct a chemical experiment on it in which the presence of nickel in the coin will be assessed (to the best of your knowledge, the probability is $\left.P_{B}(N)=\frac{9}{10}\right)$. The choice is yours. The probabilities are given by two measures, $P_{A}$ and $P_{B}$. Suppose you would like somehow to combine them into a single measure $P$, saying that $P_{A}(H)$ is to be understood as "the probability of the coin landing heads given that I decide to toss it", in other words, as $P(H \mid A)$. The problem is that your new measure has to ascribe probability to the event $A$ itself; in a case such as this one, when occurrence of $A$ depends on your choice, one can consider it unwise to think of $A$ having any probability whatsoever.

It turns out we encounter a similar problem when describing the Bell-type experiments. From now on, let " $L_{i}^{+}$" be the event "the measurement of the spin in direction $i$ of the particle hitting the left detector yielded the result 'up"'. The quantum mechan-

2 We use this term as meaning "different from both 0 and 1". 
ical probabilistic algorithm yields numbers naturally interpreted as probabilities in small spaces labeled by the directions of spin measurement chosen at both (or just one) detectors. For example, $P_{13}\left(L_{1}^{+} \wedge R_{3}^{+}\right)$is the probability of obtaining two "up" results at detectors set to direction 1 (the left one) and 3 (the right one); as said above, this probability is equal to $\frac{1}{2} \sin ^{2}\left(\frac{\phi_{13}}{2}\right) . P_{1}\left(L_{1}^{+}\right)$is the probability of getting the "up" result at the left detector set to direction 1 . Describing the experiment in this way is called the "many spaces approach". It employs as many probability spaces as there are possible combinations of the directions to be chosen at both detectors. However, one could prefer to have a single probability space and instead of writing " $P_{i j}\left(L_{i}^{+} \wedge R_{j}^{+}\right)$", write " $P\left(L_{i}^{+} \wedge R_{j}^{+} \mid L_{i} \wedge R_{j}\right)$ ", where $L_{i}$ is the event that the direction $i$ has been chosen at the left detector, and similarly for $R_{j}$. This-the "big space approach"-is frequently encountered in the literature regarding the connection between the PCC and the Bell inequalities (see e.g. van Fraassen (1982) or Hofer-Szabó (2008)). It, however, requires ascribing probabilities to choices of detector setting. This is one of the reasons for which we prefer to start with the small space approach (the other being its naturalness given QM's predictions) and will be using it in the next section.

\subsubsection{Deriving the Bell Inequalities}

Suppose from now on that there are four directions of spin measurement available at both detectors; let them belong to $I=\{1, \ldots, 4\}$. Consider the set of the values of the hidden variable to be $\left\{\lambda_{k}\right\}_{k \in K}$ for some $K$. The first assumption is frequently labeled as "No Conspiracy" ("NC"; it may be also referred to e.g. by "Hidden Autonomy"): the value of the hidden variable should not be statistically relevant for our choices of detector settings. In the small space approach, this is represented as

$$
\forall_{i, j, l, m \in I ; k \in K} P_{i j}\left(\lambda_{k}\right)=P_{i}\left(\lambda_{k}\right)=P_{j}\left(\lambda_{k}\right)=P_{l m}\left(\lambda_{k}\right)
$$

It would also be unreasonable to think that, given the value of the hidden variable, the direction chosen by us at one detector should be statistically relevant for the result of the measurement conducted at the other detector. This condition is called "Parameter Independence” (“PI”; sometimes labeled e.g. "Hidden Locality”):

$$
\begin{aligned}
\forall_{k \in K, i, j, l \in I, j \neq l} & P_{i j}\left(L_{i}^{+} \mid \lambda_{k}\right)=P_{i l}\left(L_{i}^{+} \mid \lambda_{k}\right)=P_{i}\left(L_{i}^{+} \mid \lambda_{k}\right) \\
& P_{i j}\left(R_{j}^{+} \mid \lambda_{k}\right)=P_{l j}\left(R_{j}^{+} \mid \lambda_{k}\right)=P_{j}\left(R_{j}^{+} \mid \lambda_{k}\right)
\end{aligned}
$$

(similarly for "down" results).

The last assumption at least partly shares the motivation with PI: given the value of the hidden variable, the result of the measurement at one detector should be statistically irrelevant to the result of the measurement conducted at the other detector. 
This condition is called “Outcome Independence” (“OI”):

$$
\begin{aligned}
\forall_{k \in K, i, j \in I} & P_{i j}\left(L_{i}^{+} \mid \lambda_{k} \wedge R_{j}^{+}\right)=P_{i j}\left(L_{i}^{+} \mid \lambda_{k}\right) \\
& P_{i j}\left(L_{i}^{-} \mid \lambda_{k} \wedge R_{j}^{-}\right)=P_{i j}\left(L_{i}^{-} \mid \lambda_{k}\right)
\end{aligned}
$$

(similarly for both pairs of "mixed" results and with $L$ and $R$ exchanged). Notice that OI states that each value of the hidden variable screens off the results of the experiment:

$$
\forall_{k \in K, i, j \in I} P_{i j}\left(L_{i}^{+} \wedge R_{j}^{+} \mid \lambda_{k}\right)=P_{i j}\left(L_{i}^{+} \mid \lambda_{k}\right) P_{i j}\left(R_{j}^{+} \mid \lambda_{k}\right)
$$

(similarly for all other three pairs of possible results).

PI and OI can be jointly expressed as the following condition ${ }^{3}$, known in the literature as "Factorisability":

$$
\forall_{k \in K, i, j \in I} P_{i j}\left(L_{i}^{+} \wedge R_{j}^{+} \mid \lambda_{k}\right)=P_{i}\left(L_{i}^{+} \mid \lambda_{k}\right) P_{j}\left(R_{j}^{+} \mid \lambda_{k}\right) \quad \text { (Factor.) }
$$

It turns out, as we will see, that PI, OI and NC jointly allow the derivation of the inequality

$-1 \leqslant P_{13}\left(L_{1}^{+} \wedge R_{3}^{+}\right)+P_{14}\left(L_{1}^{+} \wedge R_{4}^{+}\right)+P_{24}\left(L_{2}^{+} \wedge R_{4}^{+}\right)-P_{23}\left(L_{2}^{+} \wedge R_{3}^{+}\right)-P_{1}\left(L_{1}^{+}\right)-P_{4}\left(R_{4}^{+}\right) \leqslant 0$,

(Bell-CH)

which is falsified when the angles $\phi_{13}, \phi_{14}, \phi_{24}$ and $\phi_{23}$ are suitably chosen. Consider e.g. $\phi_{13}=\phi_{24}=\frac{3 \pi}{4}, \phi_{14}=\frac{5 \pi}{4}, \phi_{23}=\frac{\pi}{4}$ and $\phi_{i j}=0$ for any $i=j$. In this case we would get $\frac{\sqrt{2}-1}{2} \leqslant 0$, which is clearly false.

In section 3.1.5 we will present a "direct" derivation of the Bell- $\mathrm{CH}$, with an additional parameter referring to a potential weakening of the OI assumption (perhaps some of the values of the hidden variable are not perfect screeners?). In the coming section, though, we will use PI, OI and NC to arrive at the inequality in an indirect way, via a theorem of Fine (1982a).

\subsubsection{The Bell Inequalities via a Non-empirical Joint Measure}

There are several types of Bell inequalities. The Bell-CH inequality above is one of the so called Clauser-Horne inequalities, which refer to two measurement directions at each detector. A. Fine [1982a] proved a theorem to the effect that all inequalities of this type are derivable if and only if there exists a probability distribution over four-tuples of measurement results at all possible detector settings which returns the experimental probabilities as marginals. Such a distribution must of course be non-empirical,

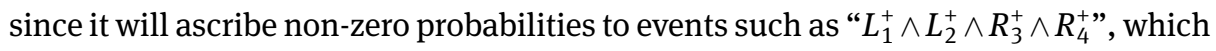

3 See Jarrett (1984) for a discussion of this point (which uses different terminology). 
are conjunctions of outcomes of measuring incompatible observables. We will show, following Fine's directions (though he used a different formalism in which the role of NC was implicit) how PI, OI and NC permit "gluing" the "small” measures $P_{1} \ldots P_{4}$ so that the appropriate "big" measure $P$ is obtained. A similar task was undertaken in Müller and Placek (2001)-however, in the context of branching models. Our considerations will not employ any additional structures.

The following is a corrected and rephrased version of Fine's theorem as presented in Müller and Placek (2001).

Theorem 2 (Fine (1982a)). Consider four probability spaces $\mathcal{L}_{i}(i \in\{1,2\}$; the event algebras $\mathcal{F}_{\mathcal{L}_{i}}$ have two atoms, $L_{i}^{+}$and $\left.L_{i}^{-}\right)$and $\mathcal{R}_{j}\left(j \in\{3,4\}\right.$; the event algebras $\mathcal{F}_{\mathcal{R}_{j}}$ have two atoms, $R_{j}^{+}$and $\left.R_{j}^{-}\right)$. Consider four measures $P_{i j}$ in the joint probability spaces with the sample space consisting of four pairs $\left\langle L_{i}^{\star}, R_{j}^{\star}\right\rangle\left(L_{i}^{\star} \in\left\{L_{i}^{+}, L_{i}^{-}\right\}, R_{j}^{\star} \in\left\{R_{j}^{+}, R_{j}^{-}\right\}\right)$ and the event algebra being the power set of the sample space. Suppose that for any $i$ and $j$ the measures $P_{i j}$ return $P_{i}$ and $P_{j}$ as marginals. Then the following conditions are equivalent:

- It is possible to define a joint probability measure $P$ on a sample space consisting of sixteen four-tuples of the shape $\left\langle L_{1}^{\star}, L_{2}^{\star}, R_{3}^{\star}, R_{4}^{\star}\right\rangle\left(L_{i}^{\star} \in\left\{L_{i}^{+}, L_{i}^{-}\right\}, R_{j}^{\star} \in\left\{R_{j}^{+}, R_{j}^{-}\right\}\right)$, with the event algebra being the power set of the sample space, in such a way that the measure returns the four joint probabilities $P_{i j}$ as marginals;

- $\quad$ The eight given probability measures satisfy the following four Bell-CH inequalities

$$
\begin{array}{r}
-1 \leqslant P_{i j}\left(L_{i}^{+} \wedge R_{j}^{+}\right)+P_{i j^{\prime}}\left(L_{i}^{+} \wedge R_{j^{\prime}}^{+}\right)+P_{i^{\prime} j^{\prime}}\left(L_{i^{\prime}}^{+} \wedge R_{j}^{++}\right)+ \\
\left.-P_{i^{\prime} j}\left(L_{i^{\prime}}^{+} \wedge R_{j}^{+}\right)-P_{i}\left(L_{i}^{+}\right)-P_{j^{\prime}}\left(R_{j^{\prime}}^{+}\right) \leqslant 0, \quad \text { (Bell-CH }\right)
\end{array}
$$

for $i, i^{\prime} \in\{1,2\} ; j, j^{\prime} \in\{3,4\}$.

To improve on clarity, instead of n-tuples and pairs we will write n-element conjunctions.

Consider first the "empirical" measures $P_{i j}$ and $P_{i}$. Enlarge the corresponding probability spaces so that the atomic events are not measurement results (or pairs of measurement results), but measurement results in conjunction with a value of a hidden variable (e.g., for some $k \in K, L_{1}^{+} \wedge \lambda_{k}$ in $\mathcal{L}_{1}$ or $L_{1}^{+} \wedge R_{3}^{+} \wedge \lambda_{k}$ in $\mathcal{L}_{13}$ ). We can therefore speak e.g. of the probability $P_{1}\left(\lambda_{k}\right)$ for any $k \in K$. The proposed measure is defined as such,

$$
P\left(L_{1}^{+} \wedge L_{2}^{+} \wedge R_{3}^{+} \wedge R_{4}^{+}\right)=\sum_{k \in K} P_{1}\left(L_{1}^{+} \mid \lambda_{k}\right) P_{2}\left(L_{2}^{+} \mid \lambda_{k}\right) P_{3}\left(R_{3}^{+} \mid \lambda_{k}\right) P_{4}\left(R_{4}^{+} \mid \lambda_{k}\right) P_{1}\left(\lambda_{k}\right),
$$

and similarly for the remaining four-tuples of possible results; each formula contains $P_{1}\left(\lambda_{k}\right)$ as its last factor. We will show that if PI, OI and NC are assumed, the measure $P$ returns the experimental probabilities as marginals. It will suffice to consider one 
case (the reasoning is analogous in other cases); let us show the following:

$$
\begin{aligned}
& P\left(L_{1}^{+} \wedge L_{2}^{+} \wedge R_{3}^{+} \wedge R_{4}^{+}\right)+P\left(L_{1}^{+} \wedge L_{2}^{+} \wedge R_{3}^{+} \wedge R_{4}^{-}\right)+P\left(L_{1}^{+} \wedge L_{2}^{-} \wedge R_{3}^{+} \wedge R_{4}^{+}\right)+ \\
& +P\left(L_{1}^{+} \wedge L_{2}^{-} \wedge R_{3}^{+} \wedge R_{4}^{-}\right)=P_{13}\left(L_{1}^{+} \wedge R_{3}^{+}\right) .
\end{aligned}
$$

The left-hand side of the equality is a sum of non-empirical probabilities, while the right-hand side is the experimental probability of two "up" results given detector settings 1 and 3.

First, notice that due to No Conspiracy we have $P_{1}\left(\lambda_{k}\right)=P_{13}\left(\lambda_{k}\right)$. Also, by employing Factorisability to each of the four elements of the above sum we can substitute $P_{13}\left(L_{1}^{+} \wedge R_{3}^{+} \mid \lambda_{k}\right)$ for $P_{1}\left(L_{1}^{+} \mid \lambda_{k}\right) P_{3}\left(R_{3}^{+} \mid \lambda_{k}\right)$. The left-hand side of (3.1) is then equal to

$$
\begin{aligned}
\sum_{k \in K} P_{13}\left(L_{1}^{+} \wedge R_{3}^{+} \mid \lambda_{k}\right) P_{13}\left(\lambda_{k}\right)\left(P_{2}\left(L_{2}^{+} \mid \lambda_{k}\right) P_{4}\left(R_{4}^{+} \mid \lambda_{k}\right)+P_{2}\left(L_{2}^{-} \mid \lambda_{k}\right) P_{4}\left(R_{4}^{+} \mid \lambda_{k}\right)+\right. \\
\left.+P_{2}\left(L_{2}^{-} \mid \lambda_{k}\right) P_{4}\left(R_{4}^{+} \mid \lambda_{k}\right)+P_{2}\left(L_{2}^{-} \mid \lambda_{k}\right) P_{4}\left(R_{4}^{+} \mid \lambda_{k}\right)\right)
\end{aligned}
$$

which after applying Factorisability to the expression in the big parentheses can be seen to equal

$$
\sum_{k \in K} P_{13}\left(L_{1}^{+} \wedge R_{3}^{+} \mid \lambda_{k}\right) P_{13}\left(\lambda_{k}\right)=P_{13}\left(L_{1}^{+} \wedge R_{3}^{+}\right)
$$

as required.

We have shown how adopting PI, OI and NC leads to the Bell inequalities in an indirect way. In the next section we will present a direct derivation.

\subsubsection{A Bell-CH Inequality from Weakened Screening Off}

It should be clear where each of the three assumptions, one of them being the requirement that the values of the hidden variable should screen off the measurement results from each other, was used in the above argument. Is it possible to derive an empirically falsifiable inequality from weaker assumptions-for example, that the values of the hidden variable are imperfect screeners, with a "margin of error" equal to some non-zero $\epsilon$ ? The answer turns out to be positive, although in this approach the margin is unfortunately close to being negligible.

Notice first that in any probability space, for any events $A, B$ and $C$ the maximal possible value of $|P(A B \mid C)-P(A \mid C) P(B \mid C)|$, intuitively understood as the inverse of the degree of "quality" of $C$ as a screener for $A$ and $B$, is $\frac{1}{4}$. The following will be our amended version of OI:

$$
\exists \epsilon \forall_{k \in K, i, j \in I} P_{i j}\left(L_{i}^{+} \wedge R_{j}^{+} \mid \lambda_{k}\right)=P_{i j}\left(L_{i}^{+} \mid \lambda_{k}\right) P_{i j}\left(R_{j}^{+} \mid \lambda_{k}\right) \pm \epsilon
$$

That is, the $\epsilon$ is the "margin of error" for all values of the hidden variable and all correlations. OI' is trivially true and is meant to be understood as a definition of $\epsilon$ in what follows. 
The derivation here proceeds using the method from Clauser and Horne (1974). The presentation is similar to the one in Placek (2000), save for introducing the $\epsilon$. The starting point is the following elementary fact:

$$
\forall_{u, u^{\prime}, v, v^{\prime} \in[0,1]}-1 \leqslant u v+u v^{\prime}+u^{\prime} v^{\prime}-u^{\prime} v-u-v^{\prime} \leqslant 0 .
$$

Now let us make the following substitutions:

$$
\begin{array}{ll}
u:=P_{13}\left(L_{1}^{+} \mid \lambda_{k}\right) ; & u^{\prime}:=P_{23}\left(L_{2}^{+} \mid \lambda_{k}\right) ; \\
v:=P_{13}\left(R_{3}^{+} \mid \lambda_{k}\right) ; & v^{\prime}:=P_{14}\left(R_{4}^{+} \mid \lambda_{k}\right) .
\end{array}
$$

Observe that, with the above substitutions, due to PI we know that

$$
\begin{array}{ll}
u=P_{14}\left(L_{1}^{+} \mid \lambda_{k}\right) ; & u^{\prime}:=P_{24}\left(L_{2}^{+} \mid \lambda_{k}\right) ; \\
v=P_{23}\left(R_{3}^{+} \mid \lambda_{k}\right) ; & v^{\prime}:=P_{24}\left(R_{4}^{+} \mid \lambda_{k}\right) .
\end{array}
$$

After taking this into account and multiplying all sides of (3.2) by $P_{13}\left(\lambda_{k}\right)$ we get

$$
\begin{aligned}
& -P_{13}\left(\lambda_{k}\right) \leqslant P_{13}\left(\lambda_{k}\right) \cdot\left(P_{13}\left(L_{1}^{+} \mid \lambda_{k}\right) P_{13}\left(R_{3}^{+} \mid \lambda_{k}\right)+P_{14}\left(L_{1}^{+} \mid \lambda_{k}\right) P_{14}\left(R_{4}^{+} \mid \lambda_{k}\right)+\right. \\
& \left.\quad+P_{24}\left(L_{2}^{+} \mid \lambda_{k}\right) P_{24}\left(R_{4}^{+} \mid \lambda_{k}\right)-P_{23}\left(L_{2}^{+} \mid \lambda_{k}\right) P_{23}\left(R_{3}^{+} \mid \lambda_{k}\right)-P_{13}\left(L_{1}^{+} \mid \lambda_{k}\right)-P_{24}\left(R_{4}^{+} \mid \lambda_{k}\right)\right) \leqslant 0 .
\end{aligned}
$$

It is now time to employ OI', so the term $\epsilon$ is introduced:

$$
\begin{aligned}
& -P_{13}\left(\lambda_{k}\right) \leqslant P_{13}\left(\lambda_{k}\right) \cdot\left(P_{13}\left(L_{1}^{+} \wedge R_{3}^{+} \mid \lambda_{k}\right) \pm \epsilon+P_{14}\left(L_{1}^{+} \wedge R_{4}^{+} \mid \lambda_{k}\right) \pm \epsilon+\right. \\
& \left.\quad+P_{24}\left(L_{2}^{+} \wedge R_{4}^{+} \mid \lambda_{k}\right) \pm \epsilon-P_{23}\left(L_{2}^{+} \wedge R_{3}^{+} \mid \lambda_{k}\right) \pm \epsilon-P_{13}\left(L_{1}^{+} \mid \lambda_{k}\right)-P_{24}\left(R_{4}^{+} \mid \lambda_{k}\right)\right) \leqslant 0 .
\end{aligned}
$$

We now use NC and multiply all expressions in the big parentheses by $P_{13}\left(\lambda_{k}\right)$ :

$$
\begin{gathered}
-P_{13}\left(\lambda_{k}\right) \leqslant P_{13}\left(L_{1}^{+} \wedge R_{3}^{+} \mid \lambda_{k}\right) P_{13}\left(\lambda_{k}\right) \pm \epsilon \cdot P_{13}\left(\lambda_{k}\right)+P_{14}\left(L_{1}^{+} \wedge R_{4}^{+} \mid \lambda_{k}\right) P_{14}\left(\lambda_{k}\right) \pm \epsilon \cdot P_{13}\left(\lambda_{k}\right)+ \\
+P_{24}\left(L_{2}^{+} \wedge R_{4}^{+} \mid \lambda_{k}\right) P_{24}\left(\lambda_{k}\right) \pm \epsilon \cdot P_{13}\left(\lambda_{k}\right)-P_{23}\left(L_{2}^{+} \wedge R_{3}^{+} \mid \lambda_{k}\right) P_{23}\left(\lambda_{k}\right) \pm \epsilon \cdot P_{13}\left(\lambda_{k}\right)+ \\
-P_{13}\left(L_{1}^{+} \mid \lambda_{k}\right) P_{13}\left(\lambda_{k}\right)-P_{24}\left(R_{4}^{+} \mid \lambda_{k}\right) P_{24}\left(\lambda_{k}\right) \leqslant 0
\end{gathered}
$$

which is by the definition of conditional probability equivalent to

$$
\begin{aligned}
-P_{13}\left(\lambda_{k}\right) \leqslant P_{13}\left(L_{1}^{+} \wedge R_{3}^{+} \wedge \lambda_{k}\right) \pm \epsilon \cdot P_{13}\left(\lambda_{k}\right)+P_{14}\left(L_{1}^{+} \wedge R_{4}^{+} \wedge \lambda_{k}\right) \pm \epsilon \cdot P_{13}\left(\lambda_{k}\right)+ \\
+P_{24}\left(L_{2}^{+} \wedge R_{4}^{+} \wedge \lambda_{k}\right) \pm \epsilon \cdot P_{13}\left(\lambda_{k}\right)-P_{23}\left(L_{2}^{+} \wedge R_{3}^{+} \wedge \lambda_{k}\right) \pm \epsilon \cdot P_{13}\left(\lambda_{k}\right)+ \\
-P_{13}\left(L_{1}^{+} \wedge \lambda_{k}\right)-P_{24}\left(R_{4}^{+} \wedge \lambda_{k}\right) \leqslant 0
\end{aligned}
$$

Let us now sum over $k \in K$. We arrive at two inequalities:

$$
\begin{aligned}
& -1 \leqslant P_{13}\left(L_{1}^{+} \wedge R_{3}^{+}\right)+P_{14}\left(L_{1}^{+} \wedge R_{4}^{+}\right)+P_{24}\left(L_{2}^{+} \wedge R_{4}^{+}\right)-P_{23}\left(L_{2}^{+} \wedge R_{3}^{+}\right)-P_{1}\left(L_{1}^{+}\right)-P_{4}\left(R_{4}^{+}\right)-4 \epsilon ; \\
& P_{13}\left(L_{1}^{+} \wedge R_{3}^{+}\right)+P_{14}\left(L_{1}^{+} \wedge R_{4}^{+}\right)+P_{24}\left(L_{2}^{+} \wedge R_{4}^{+}\right)-P_{23}\left(L_{2}^{+} \wedge R_{3}^{+}\right)-P_{1}\left(L_{1}^{+}\right)-P_{4}\left(R_{4}^{+}\right)+4 \epsilon \leqslant 0 .
\end{aligned}
$$


If $\epsilon=0$, the outcome is simply the Bell-CH inequality. Notice that if the angles between measurement directions are chosen as noted on $p$. 47, we get that $\frac{\sqrt{2}-1}{2}+4 \epsilon \leqslant 0$, which is false for $\epsilon<0.052$. This is the degree to which we can weaken the requirement of screening off present in OI and still derive a falsifiable inequality, keeping the other assumptions intact and not introducing any new assumptions. Admittedly, it is a very modest weakening.

\subsubsection{Connection with the PCC}

Since the Bell inequalities have been shown to be false, it would seem that one (at least) from the three assumptions-NC, PI or OI-must go. Although there are dissenting opinions (e.g. Stapp's arguments against locality), the majority view is that OI is the culprit. Van Fraassen [1982] was apparently the first to claim that the issue was connected with Reichenbach's ideas; the Bell setup is an example of a "conceivable phenomenon in which there is a correlation for which there can exist no common cause". Structurally, the argument proceeds by reductio; if a common cause is posited, Bell inequalities follow. And the existence of a common cause is taken to be expressed by OI-but in the big space approach (van Fraassen labels the assumption as "Causality"). Earlier (p. 100), the author generalizes the notion of a common cause to "not just a yes-no event", leaving out the statistical relevance conditions, thus arriving at the notion which we labeled as "screener system". Of course, if in the case of the Bell setup no screener systems exist (given NC and PI, which we do not want to abandon), then a fortiori no statistical common cause systems exist, too, which would seem to be against Reichenbach's idea. Since then, however, a number of authors have raised concerns that one cannot disprove Reichenbach's Principle by such arguments, because they show the nonexistence of a single common cause for all correlations (a common common cause), which the Principle does not require to exist. The situation in the literature is roughly as follows (since all the authors use their own formalisms, in the following list we will abstain from using any formalism whatsoever):

- Belnap and Szabó (1996) first note the apparent discrepancy in what the violation of Bell inequalities is taken to prove and what the PCC actually claims; an argument regarding the nonexistence of a common common cause of the EinsteinPodolski-Rosen (EPR) correlations (modally interpreted) is given in the Branching Space-Times setting; ${ }^{4}$

- Szabó (2000) presents a model for the EPR correlations in which different correlations are screened off by different common causes; however, as the author himself

4 The Branching Space-Times (BST) approach to Bell-type experiments is still being developed, see e.g. Placek (2010); however, discussing it here would not be worthwhile, since it would require introducing the BST formalism, and the conclusions do not consider the issue at hand. 
notes, the model does not satisfy a stronger (but feasible) version of No Conspiracy. Namely, detector settings may be statistically relevant for some Boolean combinations of the values of common causes; Szabó conjectures that this is inevitable and no "corrected" model can be given;

- Graßhoff et al. (2005) prove Szabó’s conjecture by providing a derivation of a Belltype inequality from separate common causes (as opposed to a common common cause); however, the derivation is made under the assumption that measurement results are perfectly anti-correlated, which is practically unverifiable;

- Hofer-Szabó (2008) observes that under the assumptions of the above mentioned derivation common common causes may be defined, and so the derivation is only reducibly separate-common-causal; the author presents an irreducibly separatecommon-causal derivation for "yes"-“no" common causes;

- independently, Portmann and Wüthrich (2007) address the faults of their previous paper and present an irreducible separate-common-causal (with the common causes building up partitions of arbitrary finite size) derivation of a Bell- $\mathrm{CH}$ inequality with the requirement that the anticorrelations be close to perfect-the upper bound ${ }^{5}$ was given as $2.689 \cdot 10^{-5}$;

- Higashi (2008) also presents a separate-common-causal derivation of a Bell-CH inequality, although this time the parameters are probabilities of detector settings (the author works in a kind of a big-space approach);

- Hofer-Szabó (2011) improves on the bound of Portmann and Wüthrich (2007) by providing a derivation of a falsifiable inequality (of the Wigner-type) with the requirement that the anticorrelations may be non-perfect to the margin of 1.73 . $10^{-2}$;

- Hofer-Szabó (2012) produces a separate-common-causal derivation of the Bell-CH inequality with the additional assumption that the screening off factors operate in a deterministic way (i.e., all relevant conditional probabilities are 0 or 1 ).

And so the move from common common causes to separate common causes has not lead so far to creation of a fully non-conspiratorial model for the Bell-type correlations which would preserve PI. But the task has not also been proven to be impossible, even if a vast majority of people interested in the topic share the conviction that it is. We will explore some more details of this in section 3.2. ${ }^{6}$

5 Meaning: the probability of a "+" result in one wing given a "+" result in the other wing, which in the case of perfect anticorrelations is equal to zero.

6 Suárez (2007) describes a few different kinds of "causal models" which are supposedly able to explain the failure of Factorisability (the author claims that the NC condition is a necessary condition for Factorisability, so he is concerned only with the failure of the latter, not the former). We will not discuss them here. They are to show that the failure of Factorisability does not exclude any sort of causal model for the correlations, but the models offered are obviously just "proofs of concept" and exhibit some controversial features, e.g. a past cause influencing both the emission event and the choice of 
Let us consider the connection between OI and the various forms of the PCC. The outcome will be a bit different depending on whether we choose the big or small space approach.

Big space approach. This is the formulation of OI in this approach:

$$
\forall_{i, j \in I, k \in K} P\left(L_{i}^{+} \wedge R_{j}^{+} \mid L_{i} \wedge R_{j} \wedge \lambda_{k}\right)=P\left(L_{i}^{+} \mid L_{i} \wedge R_{j} \wedge \lambda_{k}\right) P\left(R_{j}^{+} \mid L_{i} \wedge R_{j} \wedge \lambda_{k}\right)
$$

(and similarly for all other pairs of measurement results).

As said earlier, there is no mention of the statistical relevance conditions. Still, this could at first sight be simply a weakened version of PCC 3, generalized to a more than two-element partition. The starting point of the above mentioned arguments by Belnap, Szabó, Hofer-Szabó and Rédei could then be the observation that OI is presumed to be in the scope of an existential quantifier referring to $\Lambda$, the set of values of the hidden variable, and therefore the order of the quantifiers is in fact $\exists_{\Lambda=\left\{\lambda_{k}\right\}_{k \in K}} \forall_{i, j \in I, k \in K}$, meaning that by assuming OI we in fact assume the existence of a set of screening factors common for all correlations.

The matter looks differently, though. In this formulation screening off is not done by the values of the hidden variables, the various $\lambda_{k}$. The role of screeners is played by triples consisting of the values of hidden variables together with the choices of measurement settings on both detectors, for example, " $L_{1} \wedge R_{3} \wedge \lambda_{k}$ " for some $k \in K$. All such triples taken together do, in fact, constitute a partition of the "big” space. But the elements of this partition are screener-offs for different pairs of measurement outcomes, depending on which measurement settings go into the particular screener! ${ }^{7}$ It could be responded that this description is wrong, since what is to be screened in this case are not correlations, but conditional correlations. This, however, is simply moving the discussion to the small space approach. Recall that Reichenbach's Principle concerns correlation between events; a statement that for some $A, B, C, P(A B \mid C)>$ $P(A \mid C) P(B \mid C)$ (i.e., a statement of correlation of $A$ and $B$ conditional on $C$ ) does not fall within its scope.

Small space approach. Recall the formulation of OI in this approach:

$$
\forall_{k \in K, i, j \in I} P_{i j}\left(L_{i}^{+} \wedge R_{j}^{+} \mid \lambda_{k}\right)=P_{i j}\left(L_{i}^{+} \mid \lambda_{k}\right) P_{i j}\left(R_{j}^{+} \mid \lambda_{k}\right)
$$

(and similarly for all other pairs of measurement results).

In this formulation, the values of the hidden variables are screeners for the correlations in question. What is more, the same $\lambda_{k}$ 's screen off various correlations, with different measurement settings. However, they do so in different probability spaces. In each of these, the values of the hidden variable form partitions of the sample space, and all elements of the partition screen off one set of correlations: the one for the

detector settings or faster-than-light causation. One of the models is similar to the one from Butterfield (2007) and will be briefly described in section 3.4 .

7 For example, $L_{1} \wedge R_{3} \wedge \lambda_{k}$ screens off $L_{1}^{+}$from $R_{3}^{-}$, while $L_{2} \wedge R_{4} \wedge \lambda_{k}$ screens off $L_{2}^{+}$from $R_{4}^{-}$. 
particular measurement settings. Screening off of correlations under different measurement settings is done in a different probability space, with (at least potentially) a different measure. Therefore, once again, even if we allow for the generalization of Reichenbach's view to more than two-element partitions of sample spaces, the situation here does not fall within the scope of his Principle due to various spaces being required.

The moral of the last two paragraphs is this: it is not the formal notion of the common cause in Reichenbach's sense, even without the statistical relevance condition, but with the requirement of a partition of screeners, which is undermined by the falsifiability of inequalities derived from OI (among other assumptions).

On the other hand, there is an obvious intuitive connection with a less formal (and still Reichenbachian) view, e.g. that of PCC 1 . If we simply require that for any correlation there should exist a common cause, and in the case of the Bell-type setups we are looking for common causes among the (properties of) states of the source on emission, then of course OI says more then we require ${ }^{8}$; it posits the same common causes for all correlations. It is worthwhile, then, to distinguish between common common causes and separate common causes in this case. Most of the papers cited on the list on p. 51, while they do refer to Reichenbach's Principle:

- formally do not operate using exactly Reichenbach's notions, since they either work in various probability spaces or consider conditional correlations; but

- informally fully adhere to Reichenbach's view of requiring common causes, not common common causes, for correlations.

There is another-general-way of "saving" the PCC, via the notion of "causal completability” (see e.g. Hofer-Szabó et al. (1999)). Informally speaking (for now), if a probability space lacks a common cause for a correlation, it can always be extended to a bigger space, which preserves the measure on all "old" events, but contains a common cause for the previously unexplained correlation. Discussion of this notion and the proof of some results concerning it is one of the main topics of chapter 6 .

Lastly, let us note that the perspective outlined at the beginning of this sectionnamely: that the failure of Bell's inequalities means that at least one of the three assumptions of its derivations must be abandoned-may be misguided. This is because NC, PI and OI are not independent assumptions which stand or fall in isolation. In fact, the whole trio forms a single assumption, since there is an implicit existential quantification over the set of lambdas, the values of the hidden variable. An a bit extreme illustration of the idea would be this: suppose someone says "consider an integer $x$ such that 1) $x \leqslant 0$ and 2) $x>0$ ". Of course, there can be no such $x$, because the proposed conditions are mutually exclusive. But we do not conclude that one of them

8 Even if we use the small space approach and adopt PCC 2. 
is false and should meet with general abandonment. The situation here is different, because we have intuitive grounds to believe that NC and PI should hold. Still, the failure of the Bell inequalities means that the three conditions cannot jointly hold on the set of values of the posited hidden variable. If it is non-conspiratorial and screens off the measurement results at one wing from detector settings at the other wing, then it cannot be a screener-off for all the correlations between measurement results.

\subsection{Separate Common Causal Explanations}

\subsubsection{Separate Common Causes-Contra and Pro}

Placek (2009) claimed that in the case of EPR-type correlations the distinction between (separate) common causes and common common causes is a red herring. ${ }^{9}$ According to OI, each value $\lambda_{k}$ of the hidden variable screens off the correlations (the proponents of the big space approach would say "conditional correlations"). This $\lambda_{k}$ is supposed to be a complete state of the system on emission of the two particles, typically "a different state from the quantum-mechanical pair's state", which "is assumed to be an incomplete state of the pair" (Berkovitz (2008)). Two emitted pairs in the same quantum state may be in different complete states. If this interpretation of various lambdas is adopted, then, were there different screener systems for different correlations, it would follow that a system could simultaneously be in two different complete states-and so the states would not be complete after all. I believe that this strike at the notion of separate common cause explanation hits the target only if we cling to the notion that ultimately, what screens off correlations are complete states, or-in other words-complete descriptions of objects in question. In my opinion we should not expect this to happen. I will first give an (abstract) example of how we are inclined to accept screening off by incomplete states and would not expect screening off by (more) complete states. ${ }^{10}$

Look at figure 3.1. Consider a population in which two pairs of symptoms are correlated: symptom $A$ with symptom $B$ and symptom $C$ with symptom $D$. There is no information suggesting direct causation between either $A$ and $B$ or $C$ and $D$; what is more, there is similarly no information regarding causal connections between the two pairs: neither $A$ nor $B$ are thought to be causally relevant for $C$ or $D$, and vice versa. Suppose two previously hidden genetic features are discovered (between which there is also no hint of a causal connection), which meet the requirements for a statistical

\footnotetext{
9 Similar misgivings are cited by Hofer-Szabó (2011) and attributed to an anonymous referee. 10 Of course, formally there is nothing like a "more" complete state, since states are either complete or incomplete, but the gist of the example should be obvious: in general we do not expect that by including more information in the description of the events in question we close in on "real" screeners.
} 


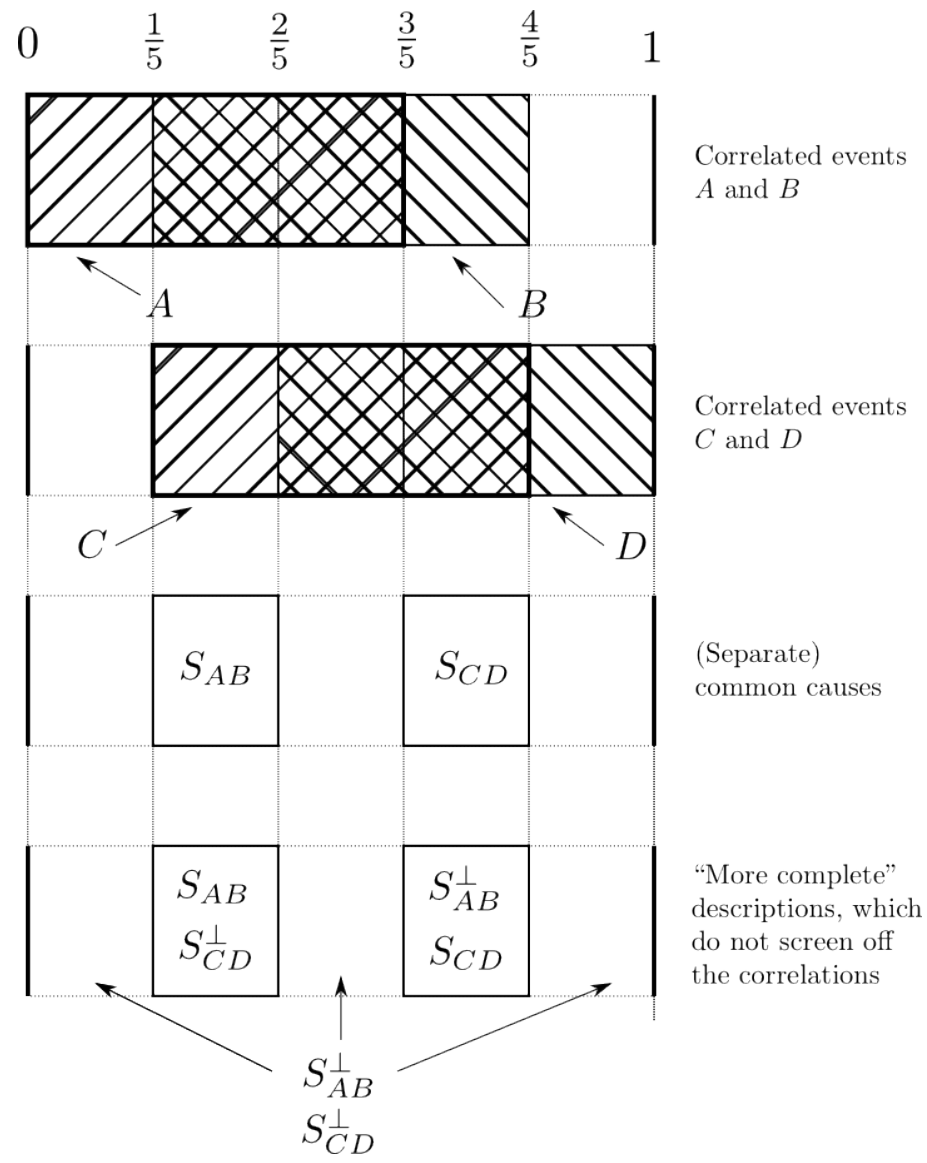

Figure 3.1: Two correlations, each screened off by an incomplete state description, but not screened off by some complete descriptions.

common cause from PCC 3: all people with the trait $S_{A B}$ display both symptoms $A$ and $B$, while the absence of the trait $S_{A B}$ makes the display of symptoms $A$ and $B$ statistically independent; similarly, all people with the trait $S_{C D}$ display both symptoms $C$ and $D$, while in the absence of the trait $S_{C D}$ the symptoms $C$ and $D$ are statistically independent. It is natural to conclude that both correlations are explained by their (separate) common causes, $S_{A B}$ in one case, $S_{C D}$ in the other. These are not complete descriptions, or complete states; suppose for clarity that other predicates are excluded and the individuals are to be described by means of possession (or lack) of the two genetic traits in question. Then, a complete state would be a Boolean combination of $S_{A B}$ and $S_{C D}$, but we would have no reason at all to expect any such combination to screen off both correlations. In the situation depicted in figure 3.1, the complete state $S_{A B}^{\perp} \cap S_{C D}^{\perp}$ screens off neither $A$ and $B$ nor $C$ and $D$. 
We can find a related, less formal example in the philosophy of biology literature, namely that concerning the levels of selection. For example, in Sober (1992) we see that the participants of the discussion consider full phenotypic descriptions to be acting as screeners. These descriptions are not "complete states" in our sense, since there are many genotypical realisations for a given phenotype. The correlations to be screened off are between survivability and possessing a certain genotype. Consider two correlations involving survivability and two genotypes, screened off by two distinct full phenotypic descriptions; in the jargon of this work we would describe the situation as displaying two separate screener systems (see definition 11, p. 35). Now, logical combinations of two distinct full phenotypic descriptions will not in general be full phenotypic descriptions (for the case similar to the one illustrated above, take the conjunction of negations of the two descriptions) and so will not be expected to act as a screening off factor in the correlations under discussion. However, it has to be observed that in this example one of the correlated factors, namely, possession of a certain genotype, is a plausible candidate for being a complete state description and in any case it will trivially screen off survivability from possessing any genotype; therefore the example is not ideal. It is still true, though, that on the level on which the participants of the discussion situate the screening factors, logical combinations of screeners are not in general screeners themselves.

For the remaining part of this section, notwithstanding the lack of a conceptual description of a separate common cause in the EPR context, I take the idea of such to be not a priori rejected, and so worth considering. I will finish by stating two open formal problems concerning the issue.

\subsubsection{Towards a Separate-common-cause Model of EPR Correlations}

To recapitulate, the issue is this: is it possible to build a probability space modelling the EPR correlations which would feature four screener systems, one for each pair of detector settings, satisfying Outcome Independence (in the sense that the screener system for a particular pair of detector settings should screen off the correlation arising at these detector settings), Parameter Independence and No Conspiracy (in the sense that no Boolean combination of elements of the screener systems should be correlated with any pair of detector settings)? Such a (hypothetical) probability space will be called a "local non-conspiratorial model with separate screener systems" ("separatess model" for short) and will be defined shortly. (Since the literature on this topic uses prevailingly the big space approach, we will adopt said approach in this section.) The question regarding the existence of such models is still open.

Suppose, then, that the role of "separate common causes" is to be played by four partitions $\left\{\lambda_{i j}^{k}\right\}_{k \in K(i j)}$; K(ij) is the index set for the screener system for the pair of detec- 
tor settings $L_{i}$ and $R_{j}$. The conditions of PI, OI, and NC are then expressed as follows ${ }^{11}$ :

$$
\begin{array}{r}
P\left(L_{i}^{m} \mid L_{i} R_{j} \lambda_{i j}^{k}\right)=P\left(L_{i}^{m} \mid L_{i} R_{j^{\prime}} \lambda_{i j}^{k}\right) \\
P\left(R_{j}^{n} \mid L_{i} R_{j} \lambda_{i j}^{k}\right)=P\left(R_{j}^{n} \mid L_{i^{\prime}} R_{j} \lambda_{i j}^{k}\right) \\
P\left(L_{i}^{m} R_{j}^{n} \mid L_{i} R_{j} \lambda_{i j}^{k}\right)=P\left(L_{i}^{m} \mid L_{i} R_{j} \lambda_{i j}^{k}\right) P\left(R_{j}^{n} \mid L_{i} R_{j} \lambda_{i j}^{k}\right) \\
P\left(L_{i} R_{j} \mathfrak{A}\right)=P\left(L_{i} R_{j}\right) P(\mathfrak{A})
\end{array}
$$

where $\mathfrak{A}$ is any Boolean combination of the elements of four partitions $\left\{\lambda_{i j}^{k}\right\}(i=$ $1,2, j=3,4)$ and all formulas are quantified for all $i \in\{1,2\}, j \in\{3,4\}, m, n \in$ $\{+,-\}$ and $k \in K(i j)$. We will not require a screener system for one correlation to satisfy Parameter Independence (and Outcome Independence) with respect to another correlation.

Definition 13[local non-conspiratorial separate-ss (common-ss) models] Consider a probability space $\langle\Omega, \mathcal{F}, P\rangle$ with events $L_{i}$ and $R_{j}$ corresponding to detector settings, and $L_{i}^{m}$ and $R_{j}^{n}$ corresponding to measurement events $(i \in\{1,2\}, j \in\{3,4\}$, $m, n \in\{+,-\})$. Suppose that the model exhibits the EPR correlations.

The probability space is a local non-conspiratorial model with separate screener systems (or a separate-ss model) for the EPR correlations if there exist four partitions of $\Omega,\left\{\lambda_{i j}^{k}\right\}_{k \in K(i j)}$, one for each pair of detector settings, such that for each such a pair $L_{i}, R_{j}$ the partition $\left\{\lambda_{i j}^{k}\right\}_{k \in K(i j)}$ meets the conditions of Parameter Independence $\left(\mathrm{PI}^{s}\right)$, Outcome Independence $\left(\mathrm{OI}^{S}\right)$ and No Conspiracy $\left(\mathrm{NC}^{S}\right)$ with regard to the correlations arising at the detector settings $L_{i}$ and $R_{j}$.

The model is deterministic if, in addition, for any $i, j$ and for any $k \in K(i j)$, the probabilities $P\left(L_{i}^{m} \mid L_{i} R_{j} \lambda_{i j}^{k}\right)$ and $P\left(R_{j}^{n} \mid L_{i} R_{j} \lambda_{i j}^{k}\right)$ are equal to 0 or 1 .

The probability space is a local non-conspiratorial model with a common screener system (or a common-ss model) for the EPR correlations if there exists a single partition of $\Omega,\left\{\lambda^{k}\right\}$, which meets the conditions of $\mathrm{OI}^{s}, \mathrm{PI}^{s}$ and $\mathrm{NC}^{s}$ with regard to all of those correlations.

The model is deterministic if, in addition, for any $i, j$ and for any $k$ the probabilities $P\left(L_{i}^{m} \mid L_{i} R_{j} \lambda^{k}\right)$ and $P\left(R_{j}^{n} \mid L_{i} R_{j} \lambda^{k}\right)$ are equal to 0 or 1 .

Notice that a common-ss model is, trivially, a separate-ss model (each correlation has its own screener system and these just happen to coincide).

\subsubsection{Bell Inequalities as Proof of Nonexistence}

The usual strategy of showing that the EPR correlations cannot be modelled in a certain way (e.g. by introducing a single screening off local hidden variable) is to directly

11 Remember that we frequently omit the “ $\cap$ ” sign: e.g. $L_{i} R_{j} \lambda_{i j}^{k}$ stands for $L_{i} \cap R_{j} \cap \lambda_{i j}^{k}$. 
derive the Bell inequalities from the probabilistic assumptions true in the purported probability space (in the small space approach we would of course have to be talking about spaces in the plural). I have already mentioned in section 3.1.6 that this feat has not been accomplished so far. But it might be interesting to consider an alternative method, which could be called a "meta-model" strategy.

The strategy goes like this: suppose a set of conditions on a probability space $P$ is presented and we would like to prove that such a space cannot exist; we are, however, unable to derive the Bell inequalities from the assumptions about $P$. We could instead try to show that if $P$ existed, then some other $P^{\prime}$, also modelling the EPR correlations, would exist, in which the Bell inequalities were derivable. Then, by contraposition, since for correlations violating the Bell inequalities $P^{\prime}$ does not exist, in such cases $P$ does not exist too.

Now, we already know that no local non-conspiratorial common-ss model for the EPR correlations exists, since the Bell inequalities are derivable from the three assumptions. By the "meta-model" strategy, to disperse any hope of a local nonconspiratorial separate-ss model it would be enough to show that any model of this kind can be transformed into a model of the common-ss kind. Tomasz Placek and me undertook this task in 2011; so far, we have been unable to obtain this result, reaching only two partial results, one of which goes in the opposite direction, namely that of proposing a certain model for the correlations.

The goal was to prove that if there is a local non-conspiratorial separate-ss model for some family of EPR correlations, then there is a local non-conspiratorial commonss model for the same correlations; in other words, that the switch to separate screener systems does not provide greater generality. We have not achieved this goal; in fact, what we arrived at is a general method for creating non-conspiratorial common-ss models for EPR correlations, which may not satisfy Parameter Independence. One way of looking at this is that, since common-ss models are trivially separate-ss models, it connects nicely with the results from Szabó (2000). With regard to the possibility of models for EPR correlations involving separate screener systems, the situation is as follows:

- Szabó (2000) produces models which satisfy $\mathrm{OI}^{s}$ and $\mathrm{PI}^{s}$, but may fail to satisfy $\mathrm{NC}^{s}$;

- in Placek and Wroński (2011) we produce models which satisfy $\mathrm{OI}^{s}$ and $\mathrm{NC}^{s}$, but may fail to satisfy $\mathrm{PI}^{s} .^{12}$

12 The details of the construction, too big to fit in this chapter, are given in the proof of Lemma 5 in Placek and Wroński (2011). The Lemma itself unfortunately claims that what is shown is the abovementioned transformation of a local non-conspiratorial separate-ss models into local non-conspiratorial common-ss models. This is not true; the proof contains a mistake-the final models may not satisfy $\mathrm{PI}^{s}$. Together with T. Placek I would like to thank Samuel Portmann and Adrian Wüthrich for finding this mistake and discussing it with us. 
Perhaps it is possible to somehow combine the two constructions and arrive at a proper local non-conspiratorial separate-ss model; I would like to leave it as problem 2 at the end of this section. Remember that the separate-ss models the construction from Placek and Wroński (2011) produces are in fact common-ss models, so any method aiming at making sure that $\mathrm{PI}^{s}$ is satisfied would also have to change the construction so that "genuinely" separate-ss models would be produced.

Suppose one wanted to go in the opposite direction and disprove the possibility of a local non-conspiratorial separate-ss model for the EPR correlations using the "metamodel" strategy. In light of the already mentioned result from Hofer-Szabó (2012), this could be achieved by showing that any separate-ss model can be transformed into a deterministic separate-ss model. Presently, the method of making a given model deterministic is only known for common-ss models:

Fact 7 (Placek). Any local non-conspiratorial common-ss model for some family of EPR correlations can be transformed into a local non-conspiratorial deterministic commonss model.

(One can prove this fact by modifying the construction from the proof of Lemma 5 from Placek and Wroński (2011); we hope to clarify these matters in a future paper.)

We are, then, left with two open problems, leading to opposite directions:

Problem 2. Provide a method of constructing local non-conspiratorial separate-ss models for the EPR correlations.

Problem 3. Show that any separate-ss model can be transformed into a deterministic separate-ss model.

If you want to show that explaining the EPR correlations by means of separate screener systems is possible, tackle problem 2. If you want to disprove this possibility, tackle problem 3. Solving one of those will mean the other is unsolvable.

\subsection{Exploiting the Detection Loophole}

In a given run of any experiment similar to the one described at the beginning of this chapter, it may happen that only one detector "fires", or even none of them does-the detectors are inefficient. This may be attributed simply to random errors of the experimental equipment, but one could also entertain the thought that the inefficiency is due to a hidden property of the emitted particles. This is the central idea behind the so called "Prism models" (dating back to Fine (1982b)).

A model of this kind is given by a meticulous construction in Szabó and Fine (2002). The hidden variable may take one of 48 values. It is deterministic in the sense 
that each value of the hidden variable predetermines whether the given particle will be detected by the detector and, if this happens, what the measurement result will be. Experimental probabilities are recovered. The main trick is a sort of "unfair sampling”, introduced so that the detected particles violate Bell-CH inequalities.

Since the model is deterministic, Factorisability (and a fortiori OI) has to hold in it. However, such models are understandably generally considered "ad hoc" (Shimony (2009)) and, with the increasing efficiency of detectors, methods were proposed for closing the detection loophole (see again Shimony (2009)).

\subsection{Common Causes as Hypersurfaces}

Lastly, let us briefly mention another PCC-related option present in the literature. Up to now, both in the big space and small space approach, the supposed common causes for Bell-type correlations were events in probability spaces. Butterfield (2007) describes in detail a view (developed first in Butterfield (1989)) called "Stochastic Einstein Locality" (SEL), in which common causes are factors which determine probabilities of events (while nothing is said about the probabilities of the factors themselves). For an event $E$ (associated with a space-time region), any hypersurface $t$ cutting through $E$ 's past light cone $C^{-}(E)$ determines a probability, $P_{t}(E)$, of $E$ 's occurrence.

The author discusses a few formulations of the SEL idea and provides proofs of their formal relations. In one version, if $E$ and $F$ are space-like related events (none is in either past or future light cone of the other) and $t$ is a hypersurface cutting through $\left(C^{-}(E) \cup C^{-}(F)\right) \backslash\left(C^{-}(E) \cap C^{-}(F)\right)$, then

$$
P_{t}(E \wedge F)=P_{t}(E) P_{t}(F) \cdot{ }^{13}
$$

The similarity with the screening off condition is obvious; it is just that the screener is in the subscript. SEL is also taken to be violated by violations of Bell inequalities. The conceptual difference between SEL and the PCC is that while the PCC (at least in its more formal shapes, like PCC 3) is plausibly falsified by everyday examples (sea levels vs. bread prices etc.) it takes a Bell-type experimental setting to violate SEL.

\subsection{Summary}

The violation of Bell inequalities has an impact on these formulations of the PCC which require screening off. If the only candidates for common causes in this case are complete states of the source on emission, then-if they are not statistically relevant for the choices of detector settings and they in turn are not statistically relevant for the

13 We omit the subscript referring to a possible world. 
measurement results in the other wing-the common causes cannot act as screeners, thus violating the first condition in the definition of a statistical common cause. This is one of the motivations for abandoning the general requirement of screening off from the definition of common causes, even if one would like to preserve Reichenbach's idea that common causes for correlations should exist (i.e. PCC 1)). The motivation is similar to the one given in arguments from conservation principles (see e.g. chapter 6 of Cartwright (1989) and section 1.4.1 of the current essay).

The recent results cited in section 3.1.6 suggest that the additional caution gained by the move from assuming common common causes to assuming separate common causes for the various correlations may not be enough to block derivations of empirically falsifiable Bell-type derivations. But the issue has not been ultimately resolved yet, as evidenced by the two open problems from the end of section 3.2. 